\title{
Microbunching instability study in a linac-driven free electron laser spreader beam line
}

\author{
S. Di Mitri and S. Spampinati \\ Elettra-Sincrotrone Trieste S.C.p.A., 34149 Basovizza, Trieste, Italy
}

(Received 22 August 2017; published 14 December 2017)

\begin{abstract}
Suppression of the microbunching instability is a priority for free electron lasers (FELs) whose goal is production of intense coherent radiation with very narrow spectral bandwidth. The instability can have large gain in multibend switchyard lines that connect the accelerator to multiple undulator lines. This study provides practical guidelines for switchyard optics designs that are largely immune to microbunching growth. A case study of the FERMI FEL switchyard is illustrated, resulting in an improved optics design that provides a unity microbunching gain while preserving the electron beam brightness. The analytical computation of the gain for the improved optics is supported by simulations and by experimental results.
\end{abstract}

DOI: 10.1103/PhysRevAccelBeams.20.120701

\section{INTRODUCTION}

The rapid development of very narrow-bandwidth free electron lasers (FELs) driven by linear accelerators (linacs) [1-5] has drawn the attention of the accelerator community to the possibility of spectral degradation driven by the development of the microbunching instability (MBI) [6,7] along the electron beam delivery system. This instability results from the interplay of collective effects such as longitudinal space charge (LSC) force, emission of coherent synchrotron radiation (CSR) in dipole magnets, and the energy-dispersion function in magnetic bunch length compressors. MBI growth typically appears as a large electron beam slice energy spread, with density and energy modulation at wavelengths comparable to those interested by the FEL emission. MBI gain must be limited in order to guarantee a high spectral brilliance, especially at output radiation wavelengths in EUV and soft x-ray range [8].

In FEL facilities with multiple undulator lines, the strength of the instability is reinforced in multibend switchyard lines (also referred to as "spreaders") that connect the accelerator to the individual undulator lines [9-13]. Due to the presence of several dipole magnets traversed by a high charge density beam, those switchyards can dramatically amplify residual density and energy modulations present on the electron beam at the exit of the linac. This study examines MBI growth in the accelerator and switchyard, and provides practical guidelines for switchyard optics designs that are largely immune to MBI, i.e., with unity gain of the instability. We provide a specific solution for the FERMI FEL spreader beam line that can be

Published by the American Physical Society under the terms of the Creative Commons Attribution 4.0 International license. Further distribution of this work must maintain attribution to the author(s) and the published article's title, journal citation, and DOI. easily implemented within the current space and with existing magnets.

FERMI $[1,2]$ is the externally seeded linac-driven FEL at Elettra Sincrotrone Trieste, Italy, emitting nearly fully coherent light in the wavelength ranges 20-100 and 4-20 nm, from the FEL-1 and the FEL-2 undulator lines respectively. The two FEL undulator lines are parallel, served by the same linac, and connected to it by the spreader. Both FELs are seeded by a wavelength-tunable external UV laser that is harmonically up-shifted via the high gain harmonic generation scheme [14]. In principle this scheme can produce nearly Fourier-transform limited FEL pulses. Practically speaking, the output spectral brilliance can, however, be degraded by unwanted structures in the electron beam longitudinal phase space as can result from serious MBI growth.

MBI in the FERMI linac has been continuously studied since the early stages of machine design [15,16], including very recent optical control of the EUV FEL spectrum [17]. MBI concerns led to the choice of operating FERMI both with a so-called laser heater (LH) [18,19], a tool that suppresses MBI via energy Landau damping, and in general using only one active magnetic bunch length compressor [15]. The FERMI LH usually improves the FEL spectral brilliance by up to factors 3 or more $[19,20]$. Nonetheless, there is experimental evidence $[17,21]$ of both enlargement and shot-to-shot variability of the spectral bandwidth for wavelengths $10 \mathrm{~nm}$ or shorter. Provisionally, this degradation is assigned to effects originating with residual MBI from the linac and to its further amplification in the spreader.

This paper aims to elucidate the role of the FERMI spreader on the MBI development, and is organized as follows. Two analytical models for the instability [22,23] are briefly reviewed in Sec. II, and then quantitatively benchmarked along the FERMI linac in Sec. III. Having demonstrated substantial agreement of the two models, we 
selected the one that appeared to be flexible enough to be applied to an arbitrary multibend line, such as the FERMI spreader. The analytical MBI gain for the present spreader configuration is confirmed by particle tracking runs, as shown in Sec. IV. In Sec. V we propose a modified, nearly isochronous spreader optics, which reduces the spreader gain to unity while preserving the beam's transverse emittance. These predictions are validated experimentally, as discussed in Sec. VI. Our conclusions are summarized in Sec. VII.

\section{THEORETICAL BACKGROUND}

We first consider the MBI model introduced by Huang and Kim [22] (henceforth labeled the "HK-model"). It is based on integral equations for the bunching factor, i.e., the Fourier transform of the bunch current density function:

$$
b(k ; s)=\frac{1}{N} \int d \vec{X} e^{-i k z} f(\vec{X} ; s),
$$

where $N$ is the number of particles in the bunch, $k=2 \pi / \lambda$ is the modulation wave number, $\vec{X}=\left(x, x^{\prime}, z, \delta\right)$ is the fourdimensional (4D) phase space vector, and $f(\vec{X} ; s)$ is the beam distribution function in the transverse and in the longitudinal phase space at location $s$ along the beam line. The evolution of the beam energy distribution is governed by 1D free-space, steady-state impedance models. Within a bunch compressor chicane composed of individual dipole magnets, the CSR impedance is [7]

$$
Z_{\mathrm{CSR}}(k ; s)=(0.94 i+1.63) \frac{k^{1 / 3}}{\rho(s)^{2 / 3}},
$$

where $\rho$ is the dipole bending radius. In a straight linac section the free-space LSC impedance per unit length is [24]

$$
Z_{\mathrm{LSC}}(k)=\frac{i Z_{0}}{\pi k r_{b}^{2}}\left[1-2 I_{1}\left(\frac{k r_{b}}{\gamma}\right) K_{1}\left(\frac{k r_{b}}{\gamma}\right)\right],
$$

where $Z_{0}=120 \pi, r_{b}=0.8735\left(\sigma_{x}+\sigma_{y}\right)$ is the effective electron beam radius, $\gamma$ the relativistic Lorentz factor and $I_{1}, K_{1}$ are modified Bessel functions of the first kind. The energy modulation induced by CSR and LSC impedances translates into amplified density modulation (bunching) by virtue of the chicane momentum compaction or $\mathrm{R}_{56}$ transport matrix term [25]. For an energy modulation amplitude $\Delta E / E_{1}$ accumulated at the chicane entrance, the resultant bunching factor at the exit of a single chicane is [22]

$$
b\left(k_{0}\right)=\left[b_{0}\left(k_{0}\right)-i C_{1} k_{0} R_{56} \frac{\Delta E\left(k_{0}\right)}{E_{1}}\right] e^{\frac{-\left(C_{1} k_{0} R_{5} \frac{\sigma_{E}}{E_{1}}\right)^{2}}{2}} .
$$

Here $E_{1}$ is the beam energy at the chicane, $C_{1}$ is the compression factor, and $\sigma_{\mathrm{E}}$ is the uncorrelated rms energy spread at the chicane entrance. The damping term represented by the exponential in Eq. (4) should be further modified to account for any smearing of bunching caused by a nonzero beam transverse emittance [22]. Both here and in all the following calculations the transverse density and uncorrelated energy spread profiles are presumed to be Gaussian.

The second model we consider was developed by Bosch et al. [23] (henceforth labeled the "B-model"). It is based on matrix formalism. All collective effects are described by frequency-dependent effective impedances separately calculated for linac straight sections and chicane dipole bend sections. Once each impedance is cast into a $2 \times 2$ matrix, the evolution of the beam longitudinal phase space, and in particular the gain in density and energy modulation, is evaluated by simple matrix multiplication. For example, the amplification of initial relative density modulation in a single-stage compression in the presence of upstream LSC impedance corresponds to the $\mathrm{T}_{1,1}$ term of the matrix associated to that beam line that has the form

$$
T_{I I}=\frac{\Delta I / I_{\text {out }}}{\Delta I / I_{\text {in }}}=F_{3}-\frac{i F_{1} F_{2} C_{1}^{2} e Z_{L S C} I_{0} k_{0} R_{56}}{E_{1}},
$$

where $F_{1}=\exp \left[-\frac{1}{2}\left(k_{0} C_{1} R_{56} \frac{\sigma_{E}}{E_{1}}\right)^{2}\right] . \mathrm{F}_{2}$ and $\mathrm{F}_{3}$ are similar to $\mathrm{F}_{1}$, but with different combinations of linear and quadratic power of $C_{1}$ [23]. The ratio of the final density (energy) modulation over the initial energy (density) modulation is calculated in a similar manner. The ratio final densityinitial density and final energy-initial density modulation amplitude can be directly compared to the one predicted by the HK-model. In the following, we will define the ratio of density-density modulation amplitudes as MBI "gain," and " $\triangle \mathrm{E}_{\mathrm{MBI}}$ " as the energy modulation amplitude driven by an initial density modulation.

The HK-model and the B-model have been compared by adopting a shot-noise driven initial density modulation that has no corresponding energy modulation. The initial bunching factor is taken to be

$$
b_{s n}\left(\lambda_{0}\right)=\sqrt{\frac{2 e c}{I_{0} \lambda_{0}}} .
$$

An additional indicator of the MBI strength, used for the comparison of the two models, is given by the amount of energy spread accumulated by the end of the beam line. Assuming that the final energy modulations are entirely converted into uncorrelated energy spread, the equivalent uncorrelated energy spread (rms value) from the MBI growth is calculated as the integral of the final energy modulation over all (or user-specified) frequency components [26]:

$$
\sigma_{E, \mathrm{MBI}}=\frac{1}{2 \pi \sqrt{2}} \sqrt{\int d \lambda \frac{\Delta E_{\mathrm{MBI}}^{2}(\lambda)}{\lambda}} .
$$




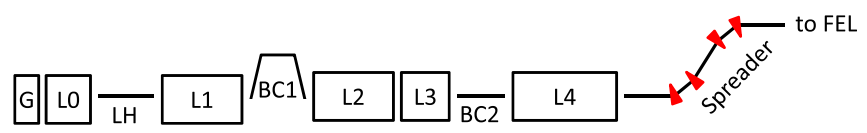

FIG. 1. Sketch, not to scale, of the FERMI linac-plus-spreader FEL-2 beam line. This study applies from the exit of L0 to the spreader end. Only the first magnetic bunch compressor, BC1, is active and for these calculations is presumed to give perfect linear compression.

\section{MBI IN THE LINAC}

The FERMI linac-plus-spreader beam line is sketched in Fig. 1. The linac is composed of multiple accelerating structures interleaved by two magnetic bunch compressors (BC1, BC2). $\mathrm{BC} 1$ only is active during standard FEL operation. Each branch of the spreader is a dog-leg made of two modified double bend achromatic cells (MDBAs), separated by quadrupole magnets [27]. Since the impact of MBI on the FEL spectrum is stronger at shorter FEL wavelengths, only that part of the spreader that feeds the FEL-2 branch line is considered in the following calculations and discussion. Table I lists typical FERMI FEL-2 operational parameters, which were adopted for the modeling of MBI in the remaining of this study.

The HK-model and the B-model both include originally CSR and LSC impedance, energy- and transverse emittance-induced Landau damping. They were further revised for the purpose of comparison as follows: (i) The LSC impedance is averaged over the transverse beam dimensions, which vary along the linac, according to the prescriptions in [24,28] [see Eq. (3)]. (ii) The MBI gain is evaluated by keeping the initial bunching factor, i.e., the low gain regime is retained together with the high gain contribution [22] [see Eq. (4)]. (iii) The effect of the LH on the electron beam initial energy distribution was calculated as in [29], assuming a laser pulse whose transverse waist size is matched at the midpoint of the LH undulator to that of the electron beam. (iv) For a fair comparison of the two models, the B-model was applied to the FERMI linac by excluding the impedance contributions of coherent edge radiation (CER), $\mathrm{LSC}$ in the $\mathrm{BC} 1$ drift sections, and the geometric longitudinal impedance of $\mathrm{rf}$ structures.

Figures 2 and 3 compare the two models' prediction, with and without LH, for the parameters listed in Table I. The maximum discrepancy of the curves is at $1 \%$ level, both for the spectral gain and the energy modulation amplitude. The initial modulation wavelength at which both the final MBI gain and the energy modulation are peaked differs by less than $3 \mu \mathrm{m}$ for the two models. Table II compares the final uncorrelated energy spread as computed by the quadratic sum of the initial one and that one induced by MBI [see Eq. (7)], for the two LH scenarios illustrated in Figs. 2 and 3.

The MBI gain curve and the accompanying MBIinduced energy spread are sensitive to the exact value
TABLE I. FERMI FEL-2 parameters adopted for the MBI study. The initial charge distribution is assumed to be flattop, and the bunch length compression in $\mathrm{BC} 1$ is a linear process (current shape is preserved). See Fig. 1 for naming of the individual linac sections.

\begin{tabular}{|c|c|c|}
\hline & Value & Units \\
\hline Bunch charge & 0.7 & $\mathrm{nC}$ \\
\hline Initial bunch duration, rms & 2.9 & ps \\
\hline Initial peak current & 70 & A \\
\hline Initial mean energy & 97 & $\mathrm{MeV}$ \\
\hline Initial uncorrelated energy spread, rms & 2 & $\mathrm{keV}$ \\
\hline Normalized emittance, rms $(\mathrm{x}, \mathrm{y})$ & 1,1 & $\mu \mathrm{m}$ \\
\hline Linac & Value & Units \\
\hline LH-induced energy modulation amplitude, rms & $0 / 10$ & $\mathrm{keV}$ \\
\hline LH drift length & 13 & $\mathrm{~m}$ \\
\hline L1 length & 24 & $\mathrm{~m}$ \\
\hline L1 final energy & 280 & $\mathrm{MeV}$ \\
\hline L1 average betatron functions $(\mathrm{x}, \mathrm{y})$ & 20,20 & $\mathrm{~m}$ \\
\hline $\mathrm{L} 2+\mathrm{L} 3+\mathrm{BC} 2$ length & 64 & $\mathrm{~m}$ \\
\hline L2 + L3 final energy & 750 & $\mathrm{MeV}$ \\
\hline $\mathrm{L} 2+\mathrm{L} 3$ average betatron functions $(\mathrm{x}, \mathrm{y})$ & 20,20 & $\mathrm{~m}$ \\
\hline L4 length & 63 & $\mathrm{~m}$ \\
\hline L4 final energy & 1500 & $\mathrm{MeV}$ \\
\hline L4 average betatron functions $(\mathrm{x}, \mathrm{y})$ & 20,20 & $\mathrm{~m}$ \\
\hline Magnetic compressor & Value & Units \\
\hline Linear compression factor at $\mathrm{BC} 1$ & 10 & \\
\hline Bunch duration after compression, rms & 0.3 & ps \\
\hline Peak current after compression & 700 & A \\
\hline Dipole bending angle & 85 & $\operatorname{mrad}$ \\
\hline Dipole length & 0.366 & $\mathrm{~m}$ \\
\hline Outer drift length & 2.65 & $\mathrm{~m}$ \\
\hline Total $\mathrm{R}_{56}$ & -40.9 & $\mathrm{~mm}$ \\
\hline Horizontal betatron function at $\mathrm{BC} 1$ entrance & 30 & $\mathrm{~m}$ \\
\hline Horizontal betatron function at $\mathrm{BC} 1$ waist & 5 & $\mathrm{~m}$ \\
\hline Horizontal alpha function at $\mathrm{BC} 1$ entrance & 5 & \\
\hline Spreader (actual/new) & Value & Units \\
\hline Dipole bending angle & 52 & mrad \\
\hline Dipole length & 0.4 & $\mathrm{~m}$ \\
\hline Total length & 35 & $\mathrm{~m}$ \\
\hline Total $\mathrm{R}_{56}$ & $680 / 1$ & $\mu \mathrm{m}$ \\
\hline Average betatron function & $14 / 12$ & $\mathrm{~m}$ \\
\hline Betatron function at spreader dipoles & 5 & $\mathrm{~m}$ \\
\hline
\end{tabular}

adopted for the beam initial uncorrelated energy spread. For a $0.3 \mathrm{keV}$ energy spread variation, the predicted MBI growth differs by $\sim 10 \%$ for the two models. Since this energy spread level is typically within the experimental accuracy, we will consider the two models equivalent for any practical purpose.

\section{MBI IN THE SPREADER}

We found the B-model computationally more readily applicable for computing MBI growth in the multibend FERMI spreader beam line. In this section the MBI gain 

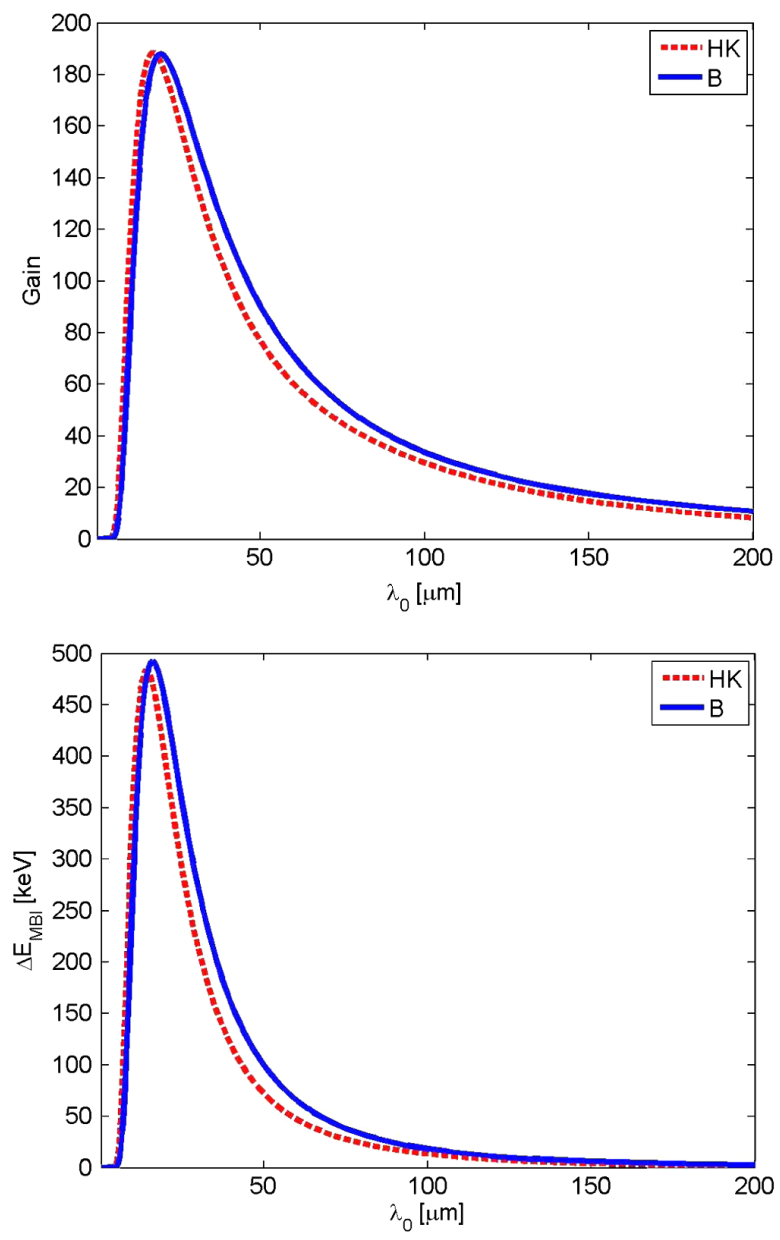

FIG. 2. Comparison of MBI gain (top) and energy modulation amplitude (bottom) at the end of the FERMI linac vs the initial density modulation wavelength. The two curves correspond to the separate predictions by the HK-model [22] (dashed line) and the B-model [23] (solid line). The laser heater is off.

through the spreader is calculated assuming no previous instability in the linac. The purpose is to identify major contributions to the gain in the spreader among different collective effects such as LSC, CSR and CER, and to benchmark the analysis with particle tracking.

The top plot of Fig. 4 presents the B-model prediction of the contribution of LSC, CSR and CER to the total gain, with the LH off. The MBI growth is dominated the by CSR impedance. The bottom plot of Fig. 4 shows the spectral dependence of the bunching factor and of the energy modulation amplitude. Dots in proximity of the analytical gain curve are from tracking runs of 30 million particles done with the ELEGANT code [30]. The simulation was set according to the prescriptions given in [31]. The numerical convergence of results was verified for number of particles in the range 1-30 million. The MBI spectral gain and $\Delta \mathrm{E}_{\mathrm{MBI}}$ are calculated from tracking results by imparting to the beam, which has a peak current of $700 \mathrm{~A}$-see Table I, an initial density modulation in the wavelength range 5-100 $\mu \mathrm{m}$ and with amplitude 1\%-10\%. Consequently,
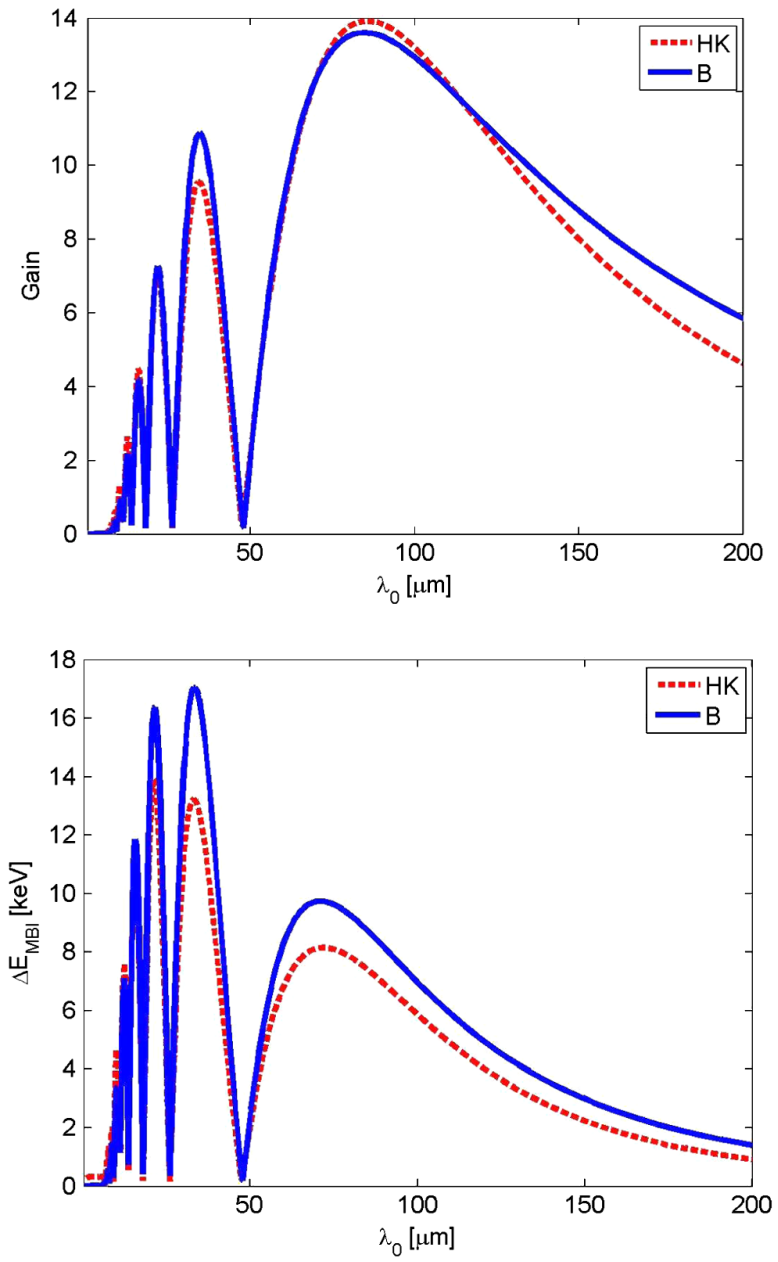

FIG. 3. Comparison of MBI gain (top) and energy modulation amplitude (bottom) at the end of the FERMI linac vs the initial density modulation wavelength. The two curves correspond to the separate predictions by the HK-model [22] (dashed line) and by the B-model [23] (solid line). For these curves, the laser heater induces $10 \mathrm{keV}$ of rms energy spread before compression.

the good agreement of the B-model with the HK-model in the linac, and with particle tracking results in the spreader, encouraged us to rely on the B-model for the study of MBI in the whole linac-plus-spreader beam line.

The development of MBI along the whole FERMI beam delivery system, now including rf impedances and CER, is illustrated in Fig. 5. The LH intensity is set to the nominal

TABLE II. Total uncorrelated energy spread (rms value) at the end of the FERMI linac (see Table I and Figs. 2 and 3). The energy modulation induced by MBI over the initial wavelength range $0.5-200 \mu \mathrm{m}$ is assumed to convert entirely into uncorrelated energy spread [see Eq. (7)].

\begin{tabular}{lccc}
\hline \hline Laser heater & HK-model & B-model & Unit \\
\hline Off & 331 & 338 & $\mathrm{keV}$ \\
$10 \mathrm{keV}$ & 102 & 110 & $\mathrm{keV}$ \\
\hline \hline
\end{tabular}



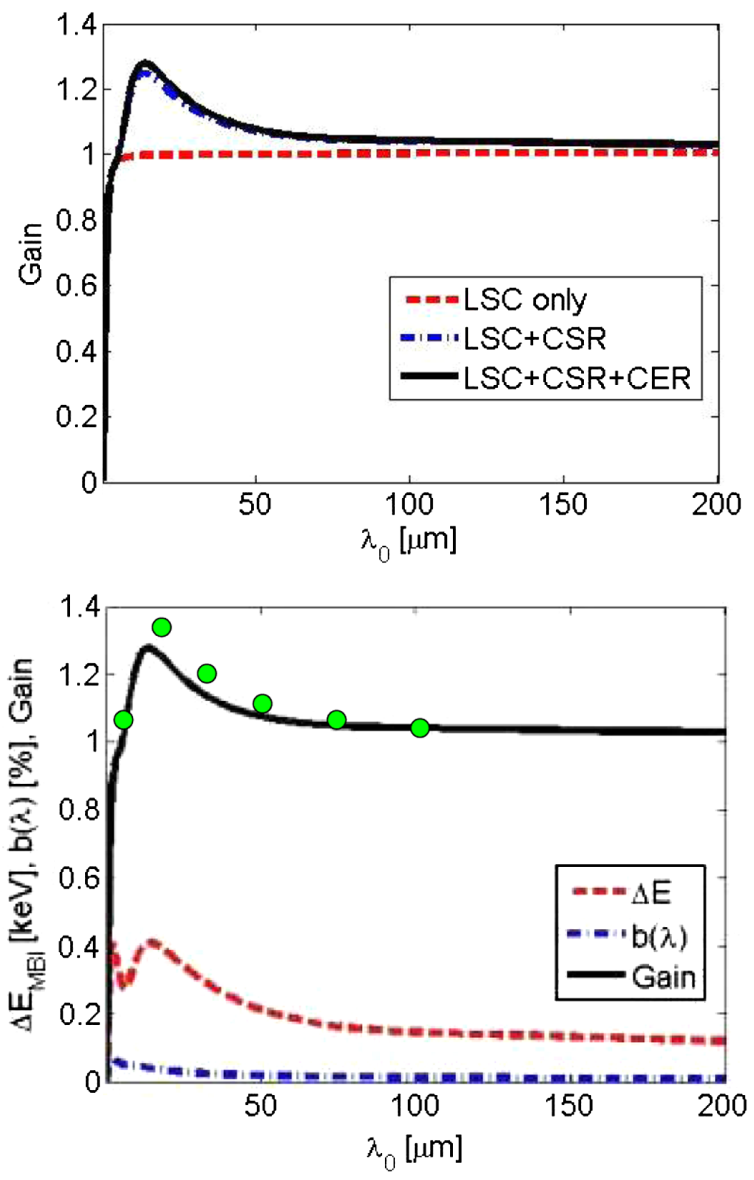

FIG. 4. Top: B-model was applied to the spreader in the presence of different collective effects and with LH off. Bottom: Spectral dependence of energy modulation amplitude $(\Delta \mathrm{E})$, bunching factor $[b(\lambda)]$ and gain through the spreader. The dots in proximity of the gain curve are from ELEGANT particle tracking runs.

$10 \mathrm{keV}$ rms energy spread level. By the end of the FERMI spreader, the total MBI gain increases by a factor up to 10 relative to that seen at the end of the linac (i.e., Fig. 3), and the energy modulation amplitudes have more than doubled (the increase is larger at shorter wavelengths). The up to $40 \mathrm{keV}$ energy modulation amplitudes accumulated at the spreader exit approach the level of the natural beam energy spread in the absence of instability; this amounts to $\sim 100 \mathrm{keV}$ and is determined by $10 \mathrm{keV}$ beam heating of the LH increased tenfold by the bunch length compression in $\mathrm{BC} 1$. Moreover, the MBI modulations lie in the final (i.e., after compression) wavelength range $0.5-5 \mu \mathrm{m}$. These wavelengths are shorter than the FERMI external seed laser pulse length (approximately $30 \mu \mathrm{m}$ long), and generally longer than the FEL-2 cooperation length (this is $0.3-0.8 \mu \mathrm{m}$ for FEL output wavelengths in the range 4-10 nm). Thus, one could expect a serious impact of such modulations on the FEL spectrum in terms of shot-toshot spectral variability and generalized bandwidth enlargement, due to excitation of sidebands on the main seeded
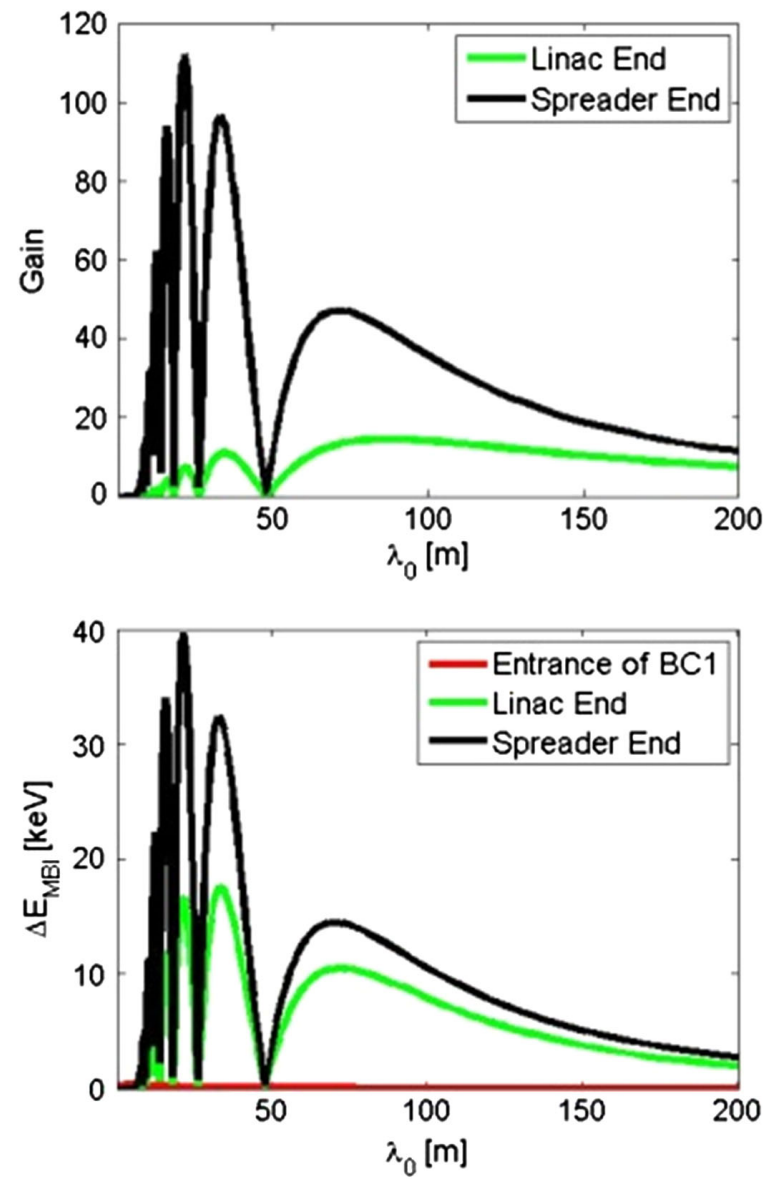

FIG. 5. Gain (top) and energy modulation amplitude (bottom), at the end of the FERMI linac (green) and of the linac-plusspreader beam line (black). The laser heater is set at $10 \mathrm{keV} \mathrm{rms}$ energy spread before compression. The bunching factor (not shown) grows up to 0.4 in the wavelength range $10-50 \mu \mathrm{m}$.

line $[16,32,33]$. These considerations fully justify serious efforts to reducing the contribution of the spreader to the global MBI dynamics, as is done in the next section.

\section{NEW SPREADER DESIGN}

The study reported in [34] suggests that a quasi-isochronous and achromatic spreader line would see a much reduced MBI gain. This suggestion was verified quantitatively for the Wisconsin FEL spreader line [35] with the help of the B-model. More recently, the simultaneous requirement of small MBI gain and preservation of the transverse beam emittance in the presence of CSR head-tail instability [36] led to the prescription of a locally isochronous beam line whose properties include small variations of $\mathrm{R}_{56}, \pi$-betatron phase advance between consecutive dipole magnets, low betatron function and relatively large alpha function at the dipoles [37] (all terms correspond to the bending plane).

These prescriptions were incorporated into a new FERMI FEL-2 spreader design, where the physical length, 

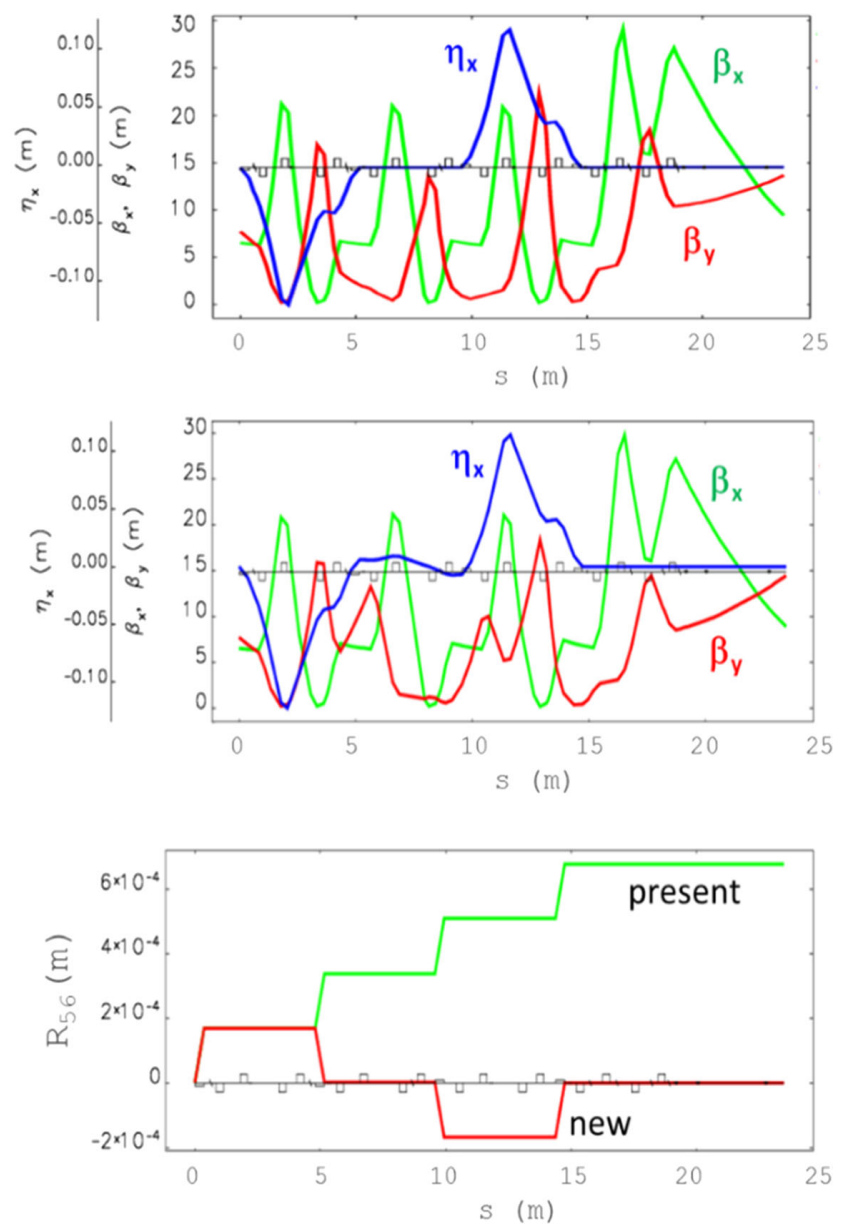

FIG. 6. Top: Linear optics functions along the FERMI spreader for the present optics design. Middle: Linear optics functions along the spreader for the new optics design. Bottom: $\mathrm{R}_{56}$ along the spreader for the present and the new optics design.

position and bending angles of the actual beam line were retained (see Table I). The total $\mathrm{R}_{56}$ of the beam line is reduced from its present value of $680 \mu \mathrm{m}$ down to $1 \mu \mathrm{m}$ (a maximum value around $5 \mu \mathrm{m}$ can be estimated on the basis of realistic magnetic errors) by virtue of the reversed sign of the dispersion function inside the two inner dipoles of the spreader branch. In spite of a small "dispersion leakage" between the two double bend cells, the whole line is still achromatic. A comparison of the present (or standard) and new linear optics functions, as well as of the $\mathrm{R}_{56}$ term along the line, is given in Fig. 6. Figure 7 replicates Fig. 5 for the new optics. As expected, the gain calculated for the new spreader optics is essentially unity at all wavelengths. The comparison shows that the new spreader design is substantially transparent to any incoming modulation induced by MBI in the linac, since the curves at the linac end (green) and at the spreader end (black) tend to superimpose, as a consequence of a unity gain through the beam line. It is worth pointing out that the new design requires only a readjustment of the strengths of the quadrupole magnets internal to the MDBAs that lies
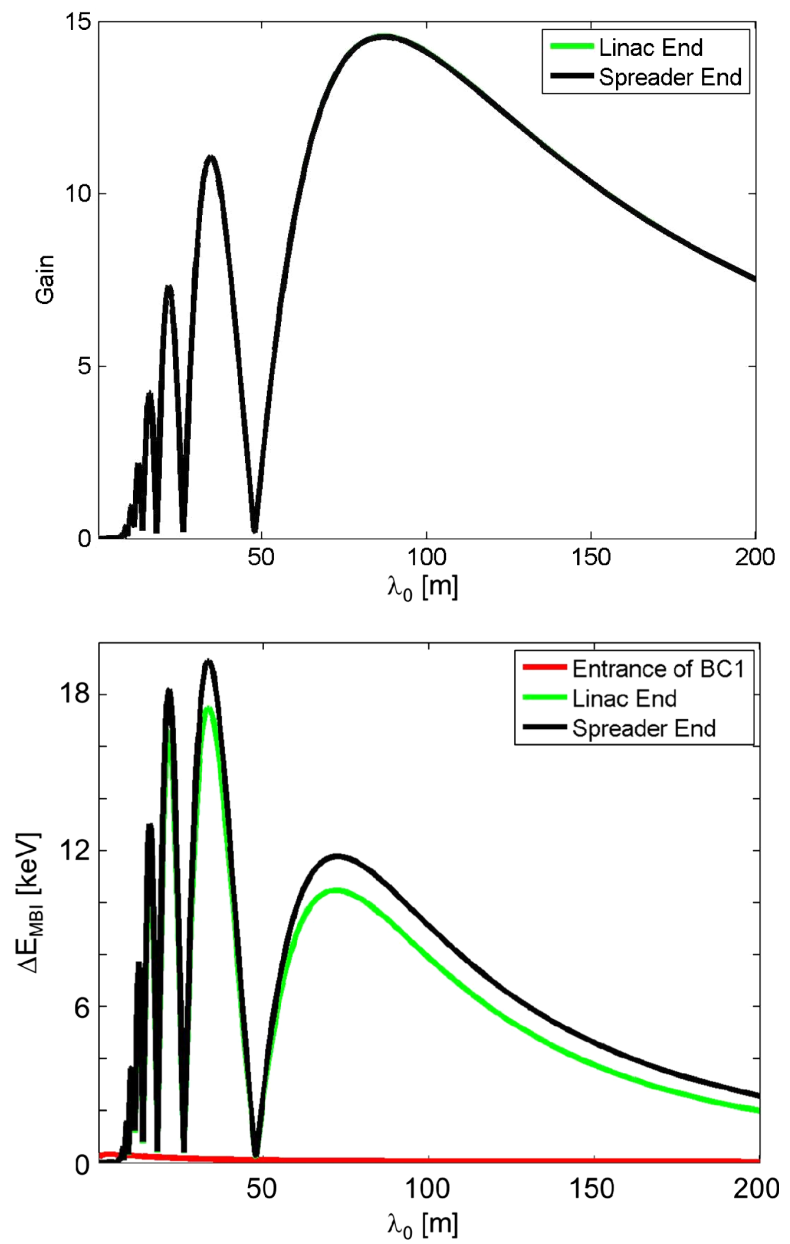

FIG. 7. Gain (top) and energy modulation amplitude (bottom) at the end of the FERMI linac (green) and linac-plus-spreader beam line (black), for the new spreader optics. The laser heater is set at $10 \mathrm{keV}$ rms energy spread before compression. The bunching factor (not shown) grows up to 0.2 in the wavelength range $10-50 \mu \mathrm{m}$. Please compare with Fig. 5.

within a range fully compatible with the FERMI operation up to $1.5 \mathrm{GeV}$ beam energy.

The present spreader optics preserves the beam horizontal normalized emittance from the CSR head-tail instability at $0.1 \mu \mathrm{m}$ level [27]. ELEGANT code tracking simulations confirm a similar performance for the new optics, in accordance to the prescription of "optics balance" [27]. In summary, by virtue of its transverse emittance preservation and unity MBI gain, the new spreader design is fully compatible with the FERMI FEL operation, and is expected to mitigate a residual effect of MBI on the FEL spectrum, especially at wavelengths shorter than $10 \mathrm{~nm}$.

\section{EXPERIMENTAL RESULTS}

The intensity of optical transition radiation (OTR) emitted by the electron beam passing through a $100 \mu \mathrm{m}$ thick-Al foil (OTR screen) at the end of the FERMI spreader was used as an indicator of the MBI gain at 


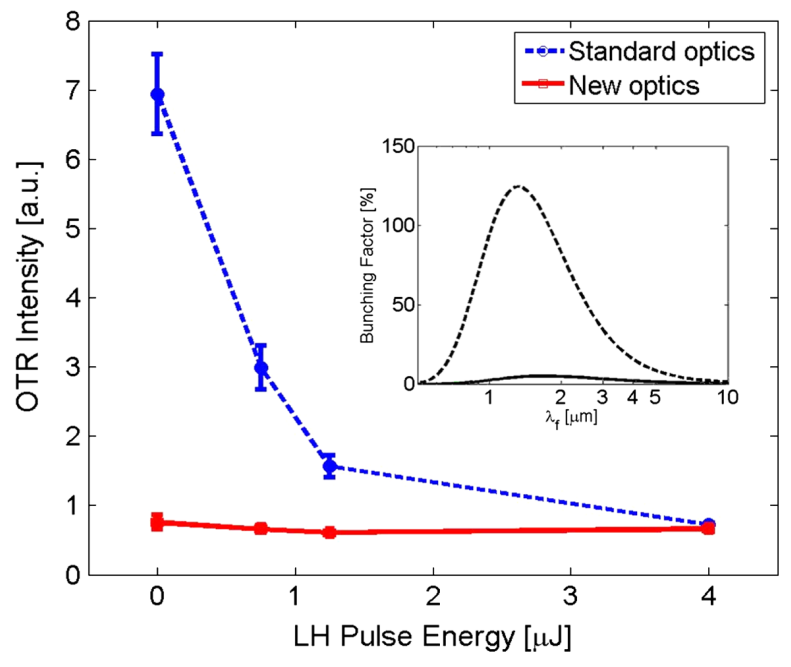

FIG. 8. OTR intensity measured at the end of the FERMI spreader vs LH pulse energy, for the standard (blue dashed) and the new optics configuration (red solid). Error bars are the rms fluctuation of the OTR signal over 500 consecutive shots. In the inset: bunching factor calculated at the end of the linac-plusspreader beam line with LH off vs final (compressed) wavelength of modulation, for the spreader standard (dashed) and new optics (solid).

optical wavelengths (the CCD of the OTR screen system is sensitive to radiation wavelengths $<3 \mu \mathrm{m}$ ), as coherent density modulations should strongly increase the OTR emission. The inset of Fig. 8 shows the reduction of the bunching factor predicted by the B-model when the new spreader optics is adopted (solid line), in comparison to the standard optics (dashed line). The main plot shows the measured OTR signal integrated over the physical region occupied by the beam spot at the screen, and averaged over many shots. The OTR signal is shown as a function of the LH pulse energy, for the standard (dashed line) and the new spreader optics (solid line). The electron beam and the linac parameters in Table I were adopted for the experiment. In both spreader optics configurations the beam sizes at the OTR screen were focused to $\sim 150 \mu \mathrm{m} \times 150 \mu \mathrm{m}$ (rms values). Control of beam sizes was accomplished by means of the last four quadrupoles of the spreader line, installed upstream of the screen and in a dispersion-free region. The above-mentioned beam sizes were measured with the $\mathrm{LH}$ set at the maximum pulse energy of $\sim 35 \mu \mathrm{J}$ in order to minimize any coherent OTR contribution to the beam image. It is worth recalling that the LH pulse energy for maximum FEL intensity is commonly between 1 and $2 \mu \mathrm{J}$, where $1 \mu \mathrm{J}$ corresponds to approximately $8 \mathrm{keV}$ of rms energy spread induced at the beam energy of $96 \mathrm{MeV}$. In accordance with the model, higher pulse energies suppress the instability at longer wavelengths [18].

The OTR intensity in the presence of the standard spreader optics shows a large sensitivity to the LH setting. A coherent OTR emission shows up as a large signal
TABLE III. Transverse rms normalized emittances measured at the entrance ("Initial") and at the exit of the FERMI spreader, for the standard and the new optics (see Fig. 6).

\begin{tabular}{lcccc}
\hline \hline & Initial & Standard optics & New optics & Unit \\
\hline$\varepsilon_{\mathrm{n}, \mathrm{x}}$ & $1.21 \pm 0.05$ & $1.29 \pm 0.05$ & $1.22 \pm 0.05$ & $\mu \mathrm{m} \mathrm{rad}$ \\
$\varepsilon_{\mathrm{n}, \mathrm{y}}$ & $1.35 \pm 0.05$ & $1.42 \pm 0.05$ & $1.41 \pm 0.05$ & $\mu \mathrm{m} \mathrm{rad}$ \\
\hline \hline
\end{tabular}

intensity for low heating level, and it disappears for $\mathrm{LH}$ energies higher than $1 \mu \mathrm{J}$. On the contrary, the OTR intensity collected in the presence of the new spreader optics is largely insensitive to the LH setting. The OTR signal value stays at the level of that one collected with the standard optics and large LH energy, $\sim 4 \mu \mathrm{J}$. No further intensity variation is observed, in both cases, at larger $\mathrm{LH}$ pulse energy (not shown).

In order to verify the simultaneous suppression of MBI and preservation of the beam transverse emittance, the emittances were measured with the quadrupole scan technique [38] at the end of the spreader. In accordance to the model [27] and to simulation results (not shown), Table III shows that the new spreader optics, as well as the standard one, preserves the horizontal emittance from CSR head-tail instability.

\section{CONCLUSIONS}

A systematic comparison of two analytical models [22,23] for MBI growth in the FERMI linac has been done, including transversally averaged LSC impedance and realistic LH effect. The two codes are found in good quantitative agreement. Given the flexibility of the B-model introduced in [23] for describing the MBI in an arbitrary transfer line, we applied it to the actual FERMI spreader beam line to FEL-2, where the code was additionally benchmarked with particle tracking runs. This study revealed a significant contribution of the spreader to the amplification of residual MBI exiting the linac, even in the situation where the FERMI laser heater is run at its nominal intensity. As a result, coherent energy modulations at the level of tens of $\mathrm{keV}$ in the final wavelength range $0.5-5 \mu \mathrm{m}$ are expected at the undulator entrance. Due to sideband formation, these modulations are expected to degrade the FEL spectral brilliance at output radiation wavelengths shorter than $\sim 10 \mathrm{~nm}$.

A new spreader optics was designed to simultaneously minimize the spreader MBI gain and preserve the horizontal beam emittance from growth associated with the CSR head-tail instability. Importantly, the new solution does not require any physical change in the existing layout and could be readily implemented. Experimental tests of the new spreader optics confirmed both the negligible MBI gain as measured by the OTR signal, and the preserved beam emittance. Consequently, we believe that this study will have an impact on the design of beam switchyard lines of existing and planned FEL facilities. 


\section{ACKNOWLEDGMENTS}

The authors acknowledge R. A. Bosch for providing, already years ago, his script for the B-model; C.-Y. Tsai for providing scripts for the analysis of MBI with ELEGANT; E. Allaria and E. Roussel for discussions on the MBI modeling and its impact on the FEL spectrum; W. M. Fawley and M. Cornacchia for reading this manuscript and helpful comments.

[1] E. Allaria et al., Highly coherent and stable pulses from the FERMI seeded free-electron laser in the extreme ultraviolet, Nat. Photonics 6, 699 (2012).

[2] E. Allaria et al., Two-stage seeded soft-x-ray free-electron laser, Nat. Photonics 7, 913 (2013).

[3] J. Amann et al., Demonstration of self-seeding in a hard-x-ray free-electron laser, Nat. Photonics 6, 693 (2012).

[4] S. Ackermann et al., Radiation at a Free-Electron Laser Directly Seeded at $38 \mathrm{~nm}$, Phys. Rev. Lett. 111, 114801 (2013).

[5] D. Ratner et al., Experimental Demonstration of a Soft X-Ray Self-Seeded Free-Electron Laser, Phys. Rev. Lett. 114, 054801 (2015).

[6] E. L. Saldin, E. A. Schneidmiller, and M. V. Yurkov, Klystron instability of a relativistic electron beam in a bunch compressor, Nucl. Instrum. Methods Phys. Res., Sect. A 490, 1 (2002).

[7] S. Heifets, G. Stupakov, and S. Krinsky, Coherent synchrotron radiation instability in a bunch compressor, Phys. Rev. ST Accel. Beams 5, 064401 (2002).

[8] S. Di Mitri and M. Cornacchia, Electron beam brightness in linac drivers for free-electron-lasers, Phys. Rep. 539, 1 (2014).

[9] W. Decking and F. Obier, Layout of the beam switchyard at the European XFEL, in Proceedings of the 11th European Particle Accelerator Conference, Genoa, 2008 (EPS-AG, Genoa, Italy, 2008), WEPC073.

[10] N. Milas and C. Gough, Design of the SwissFEL switchyard, in Proceedings of the 32nd Free Electron Laser Conference, Malmö, Sweden (Max-lab, Sweden, 2010), WEPB16.

[11] M. Placidi, J.-Y. Jung, A. Ratti, and C. Sun, Compact spreader schemes, Nucl. Instrum. Methods Phys. Res., Sect. A 768, 14 (2014).

[12] B. Faatz et al., Simultaneous operation of two soft x-ray free-electron lasers driven by one linear accelerator, New J. Phys. 18, 062002 (2016).

[13] T. Hara et al., Pulse-by-pulse multi-beam-line operation for X-ray free-electron lasers, Phys. Rev. Accel. Beams 19, 020703 (2016).

[14] L.-H. Yu, Generation of intense uv radiation by subharmonically seeded single-pass free-electron lasers, Phys. Rev. A 44, 5178 (1991).

[15] S. Di Mitri, Ph.D. thesis, University of Groningen, The Netherlands, 2011, https://www.rug.nl/research/portal/ files/14547084/10compl.pdf.
[16] S. Di Mitri et al., Design and simulation challenges for FERMI@elettra, Nucl. Instrum. Methods Phys. Res., Sect. A 608, 19 (2009).

[17] E. Roussel, E. Ferrari, E. Allaria, G. Penco, S. Di Mitri, M. Veronese, M. Danailov, D. Gauthier, and L. Giannessi, Multicolor High-Gain Free-Electron Laser Driven by Seeded Microbunching Instability, Phys. Rev. Lett. 115, 214801 (2015).

[18] E. L. Saldin, E. A. Schneidmiller, and M. Yurkov, Longitudinal space charge-driven microbunching instability in the TESLA Test Facility linac, Nucl. Instrum. Methods Phys. Res., Sect. A 528, 355 (2004).

[19] S. Spampinati et al., Laser heater commissioning at an externally seeded free-electron laser, Phys. Rev. ST Accel. Beams 17, 120705 (2014).

[20] S. Spaminati et al., Commissioning of the FERMI@Elettra laser heater, in Proceedings of the 34th International Free Electron Laser Conference, Nara, Japan (SPring-8 and Kyoto University, Japan, 2012), MOPD58.

[21] E. Allaria et al., The FERMI free-electron lasers, J. Synchrotron Radiat. 22, 485 (2015).

[22] Z. Huang and K.-J. Kim, Formulas for coherent synchrotron radiation microbunching in a bunch compressor chicane, Phys. Rev. ST Accel. Beams 5, 074401 (2002).

[23] R. A. Bosch, K. J. Kleman, and J. Wu, Modeling two-stage bunch compression with wakefields: Macroscopic properties and microbunching instability, Phys. Rev. ST Accel. Beams 11, 090702 (2008).

[24] J. Qiang, R. D. Ryne, M. Venturini, A. A. Zholents, and I. V. Pogorelov, High resolution simulation of beam dynamics in electron linacs for x-ray free electron lasers, Phys. Rev. ST Accel. Beams 12, 100702 (2009).

[25] K. L. Brown, SLAC Report No. 75, Rev. 4, UC-28, 1982.

[26] D. Ratner, C. Behrens, Y. Ding, Z. Huang, A. Marinelli, T. Maxwell, and F. Zhou, Time-resolved imaging of the microbunching instability and energy spread at the Linac Coherent Light Source, Phys. Rev. ST Accel. Beams 18, 030704 (2015).

[27] S. Di Mitri, M. Cornacchia, and S. Spampinati, Cancellation of Coherent Synchrotron Radiation Kicks with Optics Balance, Phys. Rev. Lett. 110, 014801 (2013).

[28] M. Venturini, Models of longitudinal space-charge impedance for microbunching instability, Phys. Rev. ST Accel. Beams 11, 034401 (2008).

[29] Z. Huang et al., Measurements of the linac coherent light source laser heater and its impact on the x-ray free-electron laser performance, Phys. Rev. ST Accel. Beams 13, 020703 (2010).

[30] M. Borland, Advanced Photon Source Technical Note No. LS-287, 2000.

[31] C.-Y. Tsai, Ph.D. thesis, Virginia Polytechnic Institute and State University, 2017, http://hdl.handle.net/10919/77429.

[32] D. Ratner et al., Experimental Demonstration of a Soft X-Ray Self-Seeded Free-Electron Laser, Phys. Rev. Lett. 114, 054801 (2015).

[33] Z. Zhang, R. Lindberg, W. M. Fawley, Z. Huang, J. Krzywinski, A. Lutman, G. Marcus, and A. Marinelli, Microbunching-instability-induced sidebands in a seeded free-electron laser, Phys. Rev. Accel. Beams 19, 050701 (2016). 
[34] M. Venturini and A. Zholents, Modeling microbunching from shot noise using Vlasov solvers, Nucl. Instrum. Methods Phys. Res., Sect. A 593, 53 (2008).

[35] R. Bosch, K. J. Kleman, and J. Wu, Microbunching gain of the Wisconsin FEL beam spreader, in Proceedings of the 23rd Particle Accelerator Conference, Vancouver, Canada, 2009 (IEEE, Piscataway, NJ, 2009), WE5RFP057.

[36] Ya.S. Derbenev, J. Rossbach, E. L. Saldin, and V. D. Shiltsev, Report No. TESLA-FEL 95-05, DESY, Hamburg, Germany, 1995.
[37] C.-Y. Tsai, S. Di Mitri, D. Douglas, R. Li, and C. Tennant, Conditions for coherent-synchrotron-radiation-induced microbunching suppression in multibend beam transport or recirculation arcs, Phys. Rev. Accel. Beams 20, 024401 (2017).

[38] M. G. Minty and F. Zimmermann, Report No. SLAC-R621, 2003; and in Proceedings of lectures given at the U.S. Particle Accelerator School, University of Chicago and Argonne National Laboratory, 1999. 